\title{
AVALIAÇÃO PRELIMINAR DO PARÂMETRO TERMOCINÉTICO PARA CARBONOS ATIVADOS
}

\author{
L. L. DUARTE, F. GALVÃO-LIBERATO, D. A. S. MAIA, E. VILARRASA-GARCIA, M. \\ BASTOS-NETO, D.C.S. AZEVEDO
}

Laboratório de Pesquisas em Captura de CO2/GPSA, Departamento de Engenharia Química, Universidade Federal do Ceará

E-mail para contato: lairanald@hotmail.com

\begin{abstract}
RESUMO - Em processos de separação por adsorção é necessário conhecer as características dos materiais com os quais se trabalha. Nesse contexto, as caracterizações texturais e energéticas têm um espaço importante para a maior compreensão dos materiais adsorventes, e por fim, nos processos os quais estes vão ser utilizados. Desse modo, este trabalho busca fazer uma caracterização textural de carbonos ativados produzidos a partir de polietilenotereftalato (PET) com isotermas de $\mathrm{N}_{2}$ a $77 \mathrm{~K}$, isotermas de $\mathrm{CO}_{2}$ a $273 \mathrm{~K}$ e caracterização energética com experimentos de microcalorimetria de adsorção a $298 \mathrm{~K}$, que fornece medidas diretas de calor, assim como fazer uma avaliação da cinética dos materiais analisados. A partir dos dados coletados com as análises foi ainda possível obtermos os parâmetros termocinéticos para os materiais analisados, os quais foram comparados com a distribuição de tamanho de poros obtida, estabelecendo uma relação entre os mesmos.
\end{abstract}

\section{INTRODUÇÃO}

$\mathrm{Na}$ busca por mais descobertas na área da adsorção procuram-se novas formas de caracterizar os materiais e relacionar essas características com o que acontece durante este processo. Pensando nisso, este trabalho tem por objetivo realizar um estudo preliminar dos parâmetros cinéticos para os carbonos ativados selecionados, e relacioná-los com sua distribuição do tamanho de poros obtida através de análises com $\mathrm{CO}_{2}$ a $273 \mathrm{~K}$.

Fundamenta-se a relação do parâmetro termocinético com a distribuição do tamanho de poros dos materiais, pois se sabia previamente que os materiais escolhidos são quimicamente homogêneos, ou seja, são carentes de grupo funcionais, apresentando na maior parte de sua composição carbono e hidrogênio. Dessa forma pode-se supor que a cinética do processo é regida, basicamente, pela estrutura física do material (Moura, 2017).

\section{MATERIAIS E MÉTODOS}

As amostras escolhidas para este trabalho são três carbonos ativados feitos a partir de polietilenotereftalato (PET), produzidos por Moura (2017). Essas amostras são homogêneas na sua composição química (possuem basicamente carbono e hidrogênio em sua superfície). Cada amostra é classificada pelo seu grau de burn-off, que pode ser definido como a diferença entre 
a massa inicial e a massa no fim do processo de ativação sobre massa inicial, como na Equação 1, assim, a amostra ACPX76 teria um maior grau de ativação que a ACPX41, que por sua vez teria uma maior grau de ativação que a amostra ACPX22.

$$
B O=\frac{M_{B A C O 2}-M_{A A C O 2}}{M_{B A C O 2}}
$$

Os materiais escolhidos para o trabalho foram analisados por isotermas de $\mathrm{N}_{2}$ a $77 \mathrm{~K}$, isotermas de $\mathrm{CO}_{2}$ a $273 \mathrm{~K}$ e microcalorimetria de adsorção de $\mathrm{CO}_{2}$ a $298 \mathrm{~K}$.

\subsection{Caracterização textural}

Para determinar a área superficial BET, o volume de poros, o volume de microporos e a porcentagem de microporos, as amostras foram caracterizadas com isotermas de adsorção e dessorção de $\mathrm{N}_{2}$ à $77 \mathrm{~K}$, e isotermas de $\mathrm{CO}_{2}$ à $273 \mathrm{~K}$ para a obtenção das distribuição de tamanhos de poros. Os dados de caracterização textural que serão apresentados foram obtidos por Moura (2017).

Para calcular a área superficial específica e o volume total de poros a partir dos dados experimentais Moura (2017) utilizou a equação de Brunauer-Emmett-Teller(BET) (Rouquerol et al., 2014).O volume total de poros foi estimado pela Equação 2, onde $n_{N_{2}}$ é a pressão do adsorbato a uma pressão relativa igual a $0,99, M M$ é a massa molar de $\mathrm{N}_{2}$ e $\rho_{N_{2}}$ é a densidade do $\mathrm{N}_{2}$ líquido.

$$
V_{P}=n_{N_{2}} \cdot \frac{M M_{N_{2}}}{\rho_{N_{2}}}
$$

O volume de microporos foi encontrado pelo método Dubinin-Stoeckli (DS) (Dubinin;Stoeckli, 1980) e é similar ao Dubinin-Radushkevich considerando uma superfície com diferentes estruturas porosas. A PSD pode ser estimada utilizando o método 2D Non-Local Density Functional Theory (2D NL-DFT) (Jagiello et al., 2015). O 2D NL-DFT considera a estrutura do fluido próxima a microporos estreitos e mesoporos.

\subsection{Microcalorimetria de adsorção}

Os ensaios microcalorimétricos consistem na medição simultânea da isoterma e do calor diferencial de adsorção. Os experimentos foram realizados utilizando um microcalorímetro Tian-Calvet 3D que fornece medidas diretas do calor, permitindo que sejam feitos os cálculos de entalpia de adsorção conhecendo-se a quantidade adsorvida. O equipamento é constituído de um microcalorímetro, modelo Setaram C80, acoplado a um sistema manométrico.

O procedimento consiste na injeção de doses conhecidas de gás (pressão, volume e temperatura conhecidas) em amostra regenerada, liberando uma quantidade de calor que é captada pelas termopilhas que rodeiam a célula, assim, com o sinal liberado é plotado um pico do potencial. A área desse pico multiplicada por uma constante de calibração corresponderá ao calor liberado em Joules para cada dose de pressão injetada (o software Calisto da empresa Setaram, já multiplica a área do pico pela constante de calibração e fornece o valor do calor em Joules). A quantidade adsorvida é calculada a partir de balanços de massa na fase gás. 
Simultaneamente, são recolhidos dados de equilíbrio que permitem a plotagem de uma isoterma de adsorção. O experimento foi realizado a uma temperatura de $298 \mathrm{~K}$.

A partir dos dados recolhidos, a entalpia diferencial de adsorção ( $\Delta$ hads) é calculada através da Equação 3, proposta por Rouquerol et. al (2014):

$$
\left(\frac{d Q_{r e v}}{d n^{\sigma}}\right)_{T, A}+V c\left(\frac{d p}{d n^{\sigma}}\right)_{T, A}=\Delta h_{a d s} T, n
$$

Onde dQrev é o calor medido diretamente pelo microcalorímetro, dn é o número de mols adsorvidos por ponto dosificado, Vc é o volume morto em contato com as termopilhas e dp é o diferencial de pressão para cada ponto.

\subsection{Parâmetro temocinético}

O gráfico obtido com o calor captado pelas termopilhas (potência versus tempo) fornece tanto dados termodinâmicos quanto dados cinéticos do processo, onde a cinética do calor liberado pode ser monitorada pela mudança do parâmetro termocinético.

Para a obtenção do parâmetro termocinético consideramos que o sinal calorimétrico decresce exponencialmente com o tempo a partir do ponto máximo de cada pico de adsorção. Isso pode ser aproximado pela Equação 4, onde D e Dm são o desvio no tempo t e o desvio máximo do sinal calorimétrico, respectivamente (Auroux, 2006). A partir dessa expressão, podemos calcular o parâmetro termocinético com sendo menos o inverso da inclinação da linha reta obtida plotando-se $\ln \mathrm{D}$ em função do tempo. Neste trabalho foi considerado como aceitável um $\mathrm{r}$ quadrado de pelo menos 0,97 para essa reta.

$$
D=D_{m} e^{\left(\frac{-t}{\tau}\right)}
$$

Obtidos os parâmetros termocinéticos para cada pico, é plotado um gráfico do parâmetro cinético versus pressão de equilíbrio. O tempo necessário para estabelecer o equilíbrio depende da quantidade adsorvida, da temperatura e da inércia do calorímetro.

\section{RESULTADOS E DISCUSSÃO}

\subsection{Caracterização textural}

As amostras foram caracterizadas a partir de isotermas de adsorção/dessorção de $\mathrm{N}_{2}$ a $77 \mathrm{~K}$ e de $\mathrm{CO}_{2}$ a $273 \mathrm{~K}$. A partir dos dados das isotermas de $\mathrm{N}_{2}$ foi possível calcular a área superficial, o volume total de poros e de microporos, dados que encontram-se reunidos na Tabela 1(Moura, 2017).

A partir das isotermas de $\mathrm{CO}_{2}$ a $273 \mathrm{~K}$ foram calculadas as distribuições dos tamanhos de poros. Preferiu-se utilizar os dados obtidos com $\mathrm{CO}_{2}$, pois, em comparação com a molécula de $\mathrm{N}_{2}$, a molécula de $\mathrm{CO}_{2}$ é menor o que possibilita obter resultados mais detalhados nas regiões de microporos que são maioria na estrutura dos materiais estudados. As distribuições de tamanhos de poros encontram-se reunidas na Figura 1. 
Tabela 1 - caracterização textural $\mathrm{N}_{2}$

\begin{tabular}{l|cccc}
\hline Amostra & $\begin{array}{c}\text { Area } \\
\text { Superficial } \\
\text { BET }\left(\mathrm{m}^{2} / \mathrm{g}\right)\end{array}$ & $\begin{array}{c}\text { Volume } \\
\text { de Poros } \\
\left(\mathrm{cm}^{3} / \mathrm{g}\right)\end{array}$ & $\begin{array}{c}\text { Volume de } \\
\text { Microporos } \\
\left(\mathrm{cm}^{3} / \mathrm{g}\right)\end{array}$ & $\begin{array}{c}\text { Percentagem } \\
\text { de } \\
\text { microporos }\end{array}$ \\
\hline ACPX22 & 984 & 0,405 & 0,394 & $97,3 \%$ \\
ACPX41 & 1351 & 0,585 & 0,500 & $85,5 \%$ \\
ACPX76 & 2176 & 1,035 & 0,821 & $79,3 \%$ \\
\hline
\end{tabular}

Figura 1 - Distribuição de Tamanho de Poros (PSD)
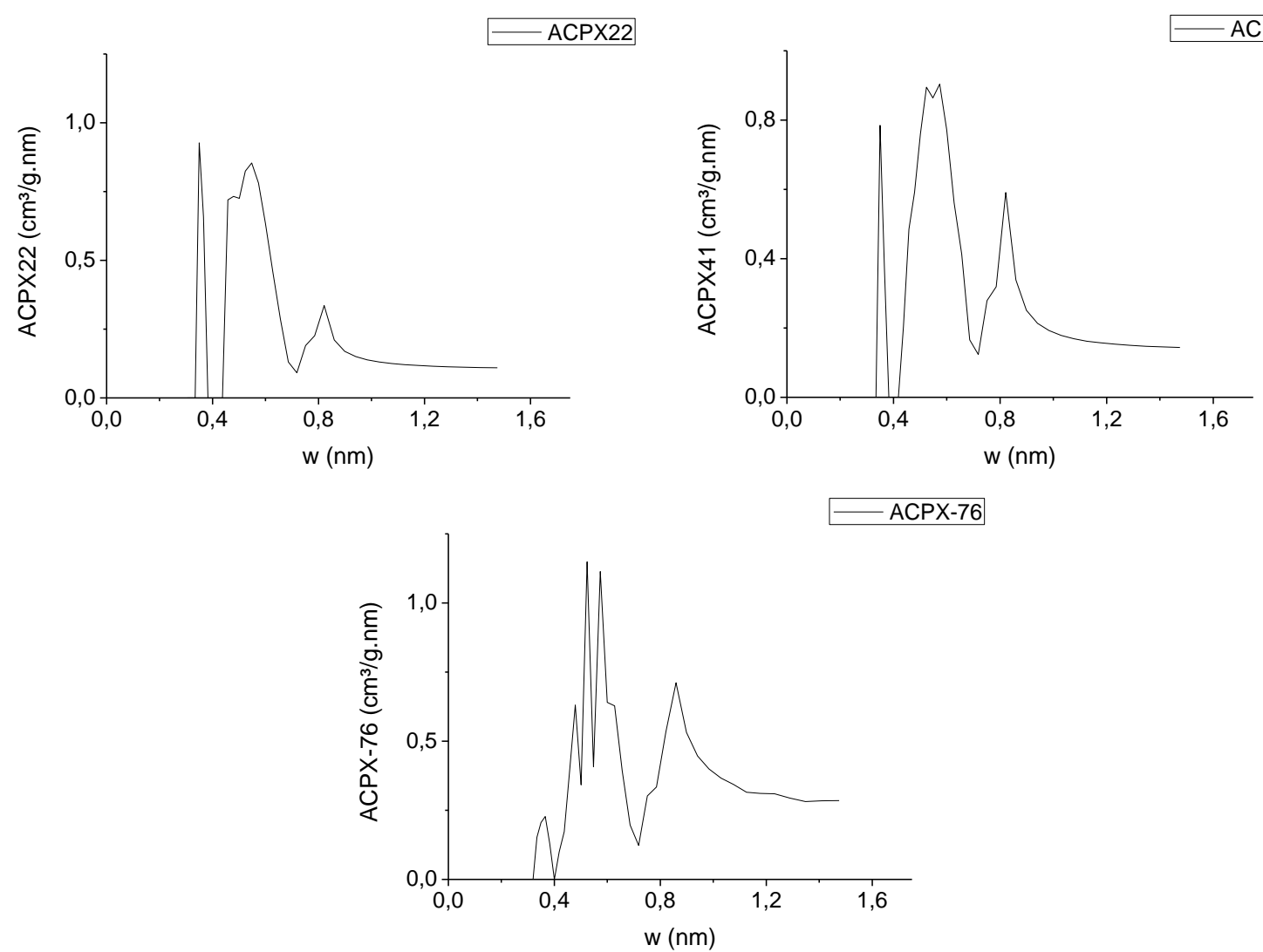

\subsection{Entalpia diferencial de adsorção}

Para estudar a energia envolvida no processo, foram feitas análises microcalorimétricas de CO2 a $298 \mathrm{~K}$. A figura 2 apresenta as curvas de entalpia diferencial obtidas.

A entalpia média para as amostras foram de 30,56 kJ para a ACPX76, 26,39 kJ para a ACPX41, e 29,12 kJ para a amostra ACPX22. Observa-se que a amostra ACPX76 apresenta uma maior diferença de energia no intervalo analisado, assim como formato característico de curvas de entalpia para materiais heterogêneos, com uma entalpia inicial de adsorção mais 
Figura 2 - Curvas de entalpia diferencial

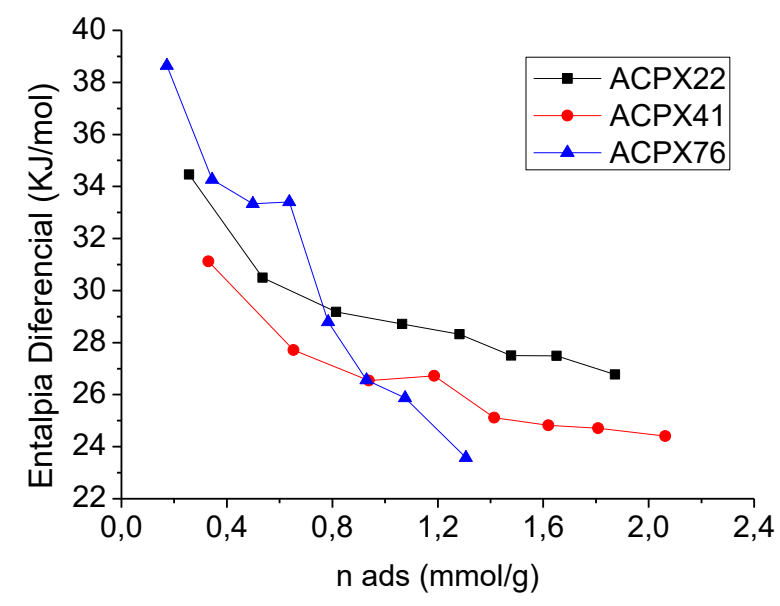

alta seguida por um degrau e uma nova queda, cada degrau apresenta a diferença entre os sítios ativos (Auroux, 2006),o que pode ser explicado por sua PSD alargada com maior diferença na proporção de tamanho dos poros da estrutura. As amostras ACPX22 e ACPX41 apresentam pequenas variações no intervalo de entalpia, o que se deve a sua distribuição de poros mais estreita, a amostra ACPX22 apresenta uma curva levemente acima da ACPX41, o que pode ser explicado pela maior porcentagem de microporos nessa amostra (Tabela1).

\subsection{Parâmetro Termocinético}

A partir dos experimentos de microcalorimetria foi possível ainda obtermos os parâmetros termocinéticos para as amostras, apresentados na Figura 3.

As curvas dos parâmetros para as amostras ACPX22 e ACPX41 apresentam valores praticamente constantes, o que é refletido em suas distribuições de tamanho de poros com formas bastante parecidas. Observe que o tempo de equilíbrio para a amostra ACPX22 é relativamente mais alto, devido à PSD mais estreita com um maior pico de volume na região de ultramicroporos $(0,4 \mathrm{~nm})$, o que aumentaria a afinidade energética por esses poros devido as forças de Van der Walls, aumentando assim o tempo de equilíbrio para essa amostra.

Já a amostra ACPX76 apresenta uma curva crescente de parâmetros termocinéticos, o que reflete a heterogeneidade dos poros encontrados na amostra devido à etapa mais longa de ativação, o que causa uma redistribuição dos poros da amostra. Perceba que, de acordo com a Figura 1, o volume de poros menores que $0,4 \mathrm{~nm}$ diminui enquanto começam a aparecer novos picos na região de $0,4-0,7 \mathrm{~nm}$ e há um grande aumento nos poros entre $0,7-1 \mathrm{~nm}$. Essa nova distribuição de volume de poros cria novos sítios energéticos que influenciam no aumento do parâmetro. Dessa forma, o tempo necessário para que se atinja o equilíbrio vai variar de acordo com o preenchimento dos poros mais energéticos, aumentando cada vez mais o tempo de equilíbrio para a amostra ACPX76, e consequentemente o valor do parâmetro termocinético. $\mathrm{O}$ longo tempo para estabelecer o equilíbrio pode ser relacionado com a distribuição das moléculas prova em centros energeticamente mais favoráveis (Auroux, 2006). 
Figura 3 - Curvas dos Parâmetros Termocinéticos

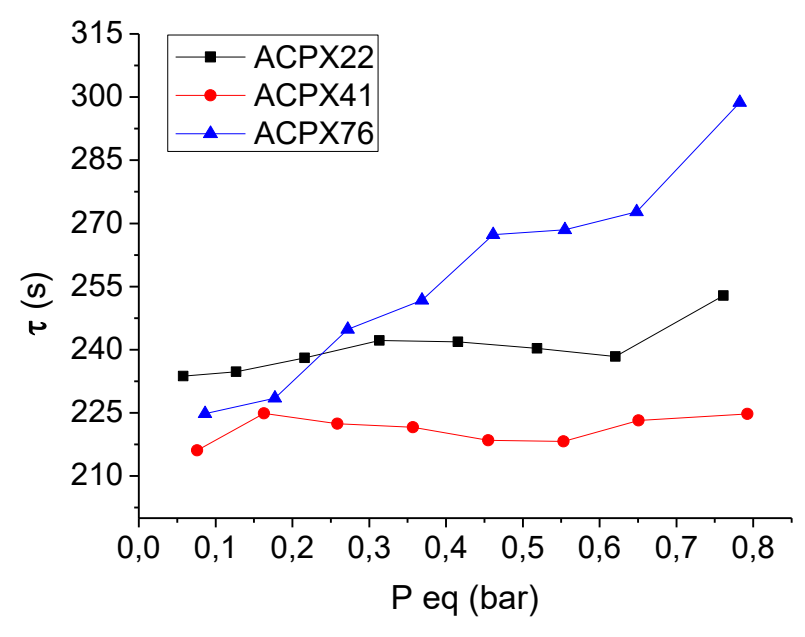

\section{CONCLUSÃO}

A partir das análises obtidas pode se concluir como estudo preliminar que os parâmetros cinéticos para as amostras ACPX22 e ACPX41 foram praticamente constantes, o que reflete a distribuição de poros parecidas das duas amostras. Já a amostra ACPX76 apresenta um parâmetro cinético crescente, o que reflete a heterogeneidade dos tamanhos de poros encontrados na amostra, devido a etapa mais longa de ativação.

\section{REFERENCIAS}

AUROUX, A. Acidity and Basicity: Determination by Adsorption Microcalorimetry. Verlag Berlin Heidelberg: Springer, 2006.

DUBININ, M. M., STOECKLI, H. F. Homogeneous and heterogeneous micropore structures in carbonaceous adsorbents. Journal of Colloid and Interface Science, 1980.

JAGIELLO, J. et al. Dual gas analysis of microporous carbons using 2D-NLDFT heterogeneous surface model and combined adsorption data of $\mathrm{N}_{2}$ and $\mathrm{CO}_{2}$. Carbon, 2015.

MOURA, P.A.S. Assessing the Potencial of Activated Carbons from Polyethylene Terephthalate (PET) as Adsorbents to Separate $\mathrm{CO}_{2}$ from Flue Gas. Master Degree dissertation: Universidade Federal do Ceará, 2017.

ROUQUEROL,F. , ROUQUEROL, J., SING, K. S. W., LLEWELLYN, P. L., MAURIN, G. Adsorption by Powder and Porous Solids(2 ${ }^{a}$ ed.). Oxford: Elsevier, 2014.

THOMMES, M. et al. Physisorption of gases, with special reference to the evaluation os surface area and pore size distribution (IUPAC Technical Report). Pure and Applied Chemistry, 2015. 